\title{
The influence of fibre length, diameter and concentration on the impact performance of long glass-fibre reinforced Polyamide 6,6.
}

\section{J. L. Thomason}

University of Strathclyde, Department of Mechanical Engineering, 75 Montrose Street, Glasgow G1 1XJ, United Kingdom.

Keywords: A Polymer-matrix composites (PMCs), B Mechanical properties, B Impact behaviour, E Injection moulding

\begin{abstract}
Results of an investigation of the mechanical performance of injection moulded long glass-fibre reinforced polyamide 6,6 composites are presented. The glass-fibre content in these composites was varied over the range of $10-50 \%$ by weight using fibres with average diameters of 10, 14 and $17 \mu \mathrm{m}$. Impact testing was carried out at $-40,23$ and $80^{\circ} \mathrm{C}$ on dry-as-moulded and boiling water conditioned samples. The results from these long fibre composites are compared with standard extrusion compounded short glassfibre materials. Data on the influence of fibre diameter, fibre concentration, residual fibre length, hydrothermal conditioning and testing temperature on the composite performance in notched and unnotched pendulum impact tests and multiaxial instrumented impact tests are presented and discussed. All of the above parameters are shown to have significant influence on impact performance. However, the level of these effects is shown to depend on which type of impact test is being considered.
\end{abstract}




\section{Introduction}

In recent years there has been strong growth in the use of long-glass-fibre thermoplastic composite systems in semi-structural and engineering applications. These thermoplastic matrix composite systems combine ease of processing with property advantages such as enhanced toughness and an unlimited shelf life. Furthermore, their intrinsic recyclability is rapidly being recognised as a strong driving force for their further application. Their potential for high-volume processing combined with high levels of end use performance and associated lower manufacturing costs has spurred the current expansion of research and development activities on thermoplastic matrix composites. Glass-fibre reinforced polyamides are excellent composite materials in terms of their high levels of mechanical performance and temperature resistance. The mechanical performance of these composites results from a combination of the fibre and matrix properties and the ability to transfer stresses across the fibre-matrix interface. Variables such as the fibre content, diameter, orientation and the interfacial strength are of prime importance to the final balance of properties exhibited by these injection moulded thermoplastic composites [17].

Short fibre reinforced thermoplastics have been used in the automotive industry for many years and there has recently been a strong growth in the use of polyamide based materials in under-the-hood applications [8]. More recently, there has been an increasing growth in the use of long fibre thermoplastic composite systems in semistructural and engineering applications. It is interesting to note that the growth rates for polypropylene based long fibre compounds has far exceeded that of other long fibre thermoplastic systems over the last decade. This has occurred despite the fact that many of the early developments and long fibre thermoplastic products were based on polyamide polymers [9-12]. It may well be that part of the background to this 
phenomenon lies in the excellent levels of profitability, processibility and performance of these materials. Achieving the correct balance of these "3P's" is critical to the success of any product in its appropriate market. Notwithstanding these facts there has been considerable discussion recently that the next major long fibre development may be in thermoplastic systems based on higher performance resins than polypropylene. Glass-fibre reinforced polyamides are excellent composite materials, however, the mechanical properties of polyamide based composites decrease markedly upon the absorption of water and other polar fluids [13-15]. There also exist a number of well documented differences in the structure-performance relationships of short fibre reinforced polyamide and polypropylene composites and it can be expected that there will also be differences when comparing these resins reinforced with long fibres.

In this report data are presented on the mechanical performance of long fibre reinforced polyamide 6,6 which may be relevant to the above discussion. Injection moulded long fibre reinforced polyamide 6,6 samples have been prepared with a range of fibre contents (0-50\% weight) and a sizing chemistry optimized for polyamide reinforcement. These long fibre compounds have been produced with glass fibres having average fibre diameters of 10, 14 and $17 \mu \mathrm{m}$. Mechanical performance has been determined for both the "dry-as-moulded" state (DaM) and after hydrolytic and temperature conditioning and compared with reference short fibre composites based on $10 \mu \mathrm{m}$ diameter fibre in the same polymer matrix. Data on the influence of the above variables on the residual fibre length and fibre orientation distribution in the moulded composites and the composite modulus and strength have been presented previously [16,17]. In this paper data on the influence of the above conditioning environments and micromechanical parameters on the composite notched and unnotched impact performance are presented and discussed. 


\section{Experimental}

The glass samples used for the production of the long glass-fibre pellets were continuous Advantex ${ }^{\circledR}$ glass (boron free E-glass) Type $30^{\circledR}$ packages produced on a single production bushing. The glass was coated with sizing formulation $\mathrm{R} 43 \mathrm{~S}$, which is a polyamide compatible sizing optimized for continuous glass products. Samples (LF10,

LF14, LF17) were produced with a range of fibre contents using nominal fibre diameters of $10,14,17 \mu \mathrm{m}$ and linear density (tex) of $1200,2400,3500 \mathrm{~g} / \mathrm{km}$ as previously described $[16,17]$. Reference short fibre compounds (SF10) were produced using DS1123 a nominal $10 \mu \mathrm{m}$ fibre diameter chopped glass product coated with polyamide sizing optimized for chopped glass production. The polyamide 6,6 (PA6,6) used for composite production was DuPont Zytel 101. Reference samples of the unreinforced resin were also included. Given that the matrix in the fibre reinforced materials experiences an extra heat cycle, reference resin samples were moulded from resin which had been run one time through the extruder with the same temperature profile as applied during the composite pellet production step.

Long fibre reinforced pellets were produced using a standard pultrusion type process [16] where the continuous glass was fed into an impregnation unit consisting of a heated oblong box containing a number of spreader bars and a circular exit die of fixed diameter. The impregnation unit was attached to, and fed by, a single screw extruder which delivered polymer melt to the unit at a rate appropriate to the pulling speed of the glass $(30 \mathrm{~m} / \mathrm{min})$ and the desired final glass:resin ratio of the pellets. The temperature of the molten resin was maintained between $300-310^{\circ} \mathrm{C}$ in the impregnation unit. After exiting the die the resin impregnated glass was cooled in a water bath before passing through a pulling and chopping operation. Nominal pellet chop length was $12.5 \mathrm{~mm}$. For the short fibre compound, the chopped glass bundles and pre-dried PA6,6 pellets were dry blended 
by weight to the appropriate glass content and compounded on a single screw extruder (2.5 inch, 3.75:1, 24:1 L/D screw). Set point temperatures were $288-293^{\circ} \mathrm{C}$ for compounding. The compounds were moulded into test bars on a 200-ton Cincinnati Milacron moulding machine. Set point temperatures were $293-299^{\circ} \mathrm{C}$ for moulding, at a mould temperature of $93^{\circ} \mathrm{C}$.

Izod and Charpy impact properties were measured on 10 specimens in accordance with the procedures in ASTM D-256 and ASTM D-4812. Instrumented impact testing was conducted using the procedures described in ASTM D3763 including a tup diameter of $12.7 \mathrm{~mm}(0.5 \mathrm{in}$.) and a supporting diameter of $38 \mathrm{~mm}$ (1.5 in.). Test samples were moulded discs of $51 \mathrm{~mm}$ ( 2 in.) diameter and $3.2 \mathrm{~mm}(0.125 \mathrm{in}$.) thickness. Tests were performed at a speed of $2.23 \mathrm{~m} / \mathrm{s}(7.33 \mathrm{ft} / \mathrm{s})$ with a drop weight of $15.9 \mathrm{~kg}(35 \mathrm{lbs})$ using an Instron Dynatup 9250HV instrumented impact tester. Unless otherwise stated, all mechanical property testing was performed at $23^{\circ} \mathrm{C}$ and at a relative humidity of $50 \%$. Fibre length, diameter, and orientation distributions were determined by image analysis and optical microscopy as previously described [16].

\section{Results}

The results for the weight average residual fibre length in the moulded composites are presented in Figure 1, where the error bars indicate the $95 \%$ confidence limits on the averages. It is clear that pultruded 'long fibre' (LF) compounds deliver significantly longer fibres to the moulded composite in comparison to the extruded short fibre (SF) compound. It is also clear that there exist significant trends for fibre length versus fibre content in Figure 1. The residual fibre length decreases strongly with increasing fibre content. As indicated in Figure 1 the data are reasonably well fit by a linear relationship with the slope of the line for the LF compounds being considerably greater than that 
observed for SF PA6,6 based compounds [13]. The data in Figure 1 also indicate that the absolute level of retained fibre length is dependent on the average fibre diameter in the composites with thinner fibres ending up shorter in the composite for any fixed value of fibre content. The dependence of the composite modulus and stiffness on the above parameters have been presented and discussed in previous papers. In summary there was no modulus dependence found on fibre length or diameter with a strong linear dependence of modulus on fibre volume fraction [16]. In contrast the strength and elongation to failure of these composites was shown to be significantly dependent on the residual fibre length, fibre diameter and fibre concentration [17].

The trends observed in notched impact for Izod and Charpy tests are shown in Figures 2 and 3. Composite notched impact is highly dependent on fibre content and exhibited a strong linear increase over the $10-30 \%$ fibre content range. Above $30 \%$ fibre content the notched impact continued to increase, however, there is some indication that the rate of increase is lower in the high fibre content region. Extrapolation of the data in Figure 2 and 3 towards zero fibre content does not intercept the value for the unreinforced resin but is much closer to a value of zero. Interestingly there are also significant differences in the PA6,6 resin value of notched impact depending on the thermal history. The value for the polymer sample which has been extruded prior to moulding, and therefore has a thermal history more comparable with the composite matrix, appears to be more in line with the composite data. There also appears to be little significant effect of fibre diameter on the notched impact of these LFPA composites, although there is some indication that the LF10 performance is slightly lower than the other samples. A highly significant observation is that the use of LF compounds gives an approximately $50 \%$ higher level of notched impact performance over the SF reference. This 'long fibre' effect is already well known to give some its greatest benefits to notched impact in PP 
based compounds where the LF/SF ratio is often in the 2-3x range [18,19], significantly greater than observed here with LFPA.

The data for notched Charpy impact at $-40^{\circ} \mathrm{C}$ in Figure 4 reveals no drop in performance of the LFPA samples in comparison to the room temperature results, whereas the PA6,6 resin and the SFPA reference sample both give significantly lower notched impact performance at $-40^{\circ} \mathrm{C}$. The results for notched Charpy impact measured at $80^{\circ} \mathrm{C}$ in Figure 5 show trends similar, in terms of fibre content, to those observed in at $-40^{\circ} \mathrm{C}$ and $23^{\circ} \mathrm{C}$. The results the PA6,6 matrix polymer alone have increased significantly due to testing above the glass transition temperature of the polymer which is in the range of $50-75^{\circ} \mathrm{C}$ depending on the measurement technique and the moisture content [20]. There is also some difference to be observed in the results in Figure 6 due to the fibre diameter. Both LF17 and LF14 notched impact have increased by a similar amount at the higher testing temperature and show clear indication of a leveling off in performance above $40 \%$ fibre content. The LF10 samples showed little change in notched impact performance at the higher testing temperature and consequently fall significantly below LF14 and LF17 in Figure 5.

The data for unnotched Izod and Charpy impact are shown in Figures 6 and 7. As has been previously observed with SFPA [13-15], the trends in unnotched impact are quite different from those in notched impact. Addition of even very small amount of reinforcement drastically reduces the unnotched performance of the PA6,6 polymer which gave 'no breaks' in both unnotched tests. In SFPA a minimum has been observed to occur in the $2-4 \% \mathrm{wt}$ range [13], all of the composites in this study have significantly higher glass contents. Consequently only increasing unnotched impact with increasing glass content is observed in the fibre content range of this study. In the case of 
unnotched Izod there is a fairly linear increase in performance, in the case of unnotched Charpy this is also the case for the LF14 and LF17 samples, however, for LF10 there is some evidence of a step increase at around $25-30 \%$ fibre content. It is also clear in Figures 6 and 7 that the fibre diameter has a significant influence on the composite unnotched impact performance, with finer fibres delivering higher unnotched impact at any fixed fibre content, across the whole range of the study. Finally it is also very interesting to note that the performance level of the SF10 reference sample in unnotched impact is not significantly different to its LF10 equivalent. Thus the effect of fibre length on PA6,6 based composite impact properties is principally an improvement in notched impact and not unnotched impact.

The data for the Charpy unnotched impact strength after boiling water conditioning presented in Figure 8 also shows dramatically different trends than the DaM data in Figure 7. The data for the LF samples all exhibit an apparently constant Charpy unnotched value of $85 \pm 5 \mathrm{~kJ} / \mathrm{m}^{2}$ with only one exception (LF10 lowest glass content sample). This level is significantly above the DaM results in Figure 7 with the greatest differences at lower fibre contents. Furthermore the SF10 reference samples show a significantly higher level of unnotched impact after conditioning than the LF10 equivalent.

The results of the instrumented impact testing are summarised in Figures 9 and 10. Figure 9 shows the energy absorbed up to the first peak in the force-time (displacement) curve, which is equivalent to the energy required to generate a critical flaw in the material. Figure 10 shows the total energy absorbed by the samples during the complete test. It is interesting to note the differences in the trends observed in Figures 9 and 10. The data for the total energy absorbed by the LFPA samples all fall approximately on a 
single trend-line which shows a strong increase in absorbed energy with increasing glass content but with little significant evidence of an effect of fibre diameter. However, the LFPA samples absorb a significantly higher level of total energy compared to the SFPA reference sample. The data for energy at first peak also show a significant dependence on fibre content. However, in contrast to the total energy data, the SFPA reference appears to give the highest performance and there is a significant effect of fibre diameter to be observed in Figure 9.

\section{Discussion}

The inverse relationship between residual fibre length and fibre diameter has been previously noted in injection moulded SFPA composites $[6,14]$ and it was further shown that the fibre aspect ratio (length/diameter) was approximately constant for samples with different fibre diameters at the same fibre content. In Figure 11 the length data from Figure 1 are considered in terms of residual fibre aspect ratio. It can be seen that the LF results all appear to collapse onto a single trend-line. However, the LF technology still delivers significantly greater aspect ratios in the moulded composites as compared to the SF samples. This is presumably related to the extrusion compounding step involved in the preparation of the SF samples and consequently the shorter fibre lengths in the SF compounds prior to injection moulding. An important issue to be considered here is the relationship between fibre volume fraction, fibre aspect ratio and fibre packing. In general it can be understood that to pack more and more fibres into a fixed volume the average level of fibre alignment must be increased and/or the fibre aspect ratio will be decreased. A number of authors [21-23] have indicated that the maximum volume fraction obtainable with fully $3 \mathrm{D}$ random fibre orientation is related to the fibre aspect ratio by the equation 


$$
V_{f} \frac{L}{D}=C
$$

where the value of the constant $\mathrm{C}$ is somewhere in the range 4-7. Equation 1 can also be used [21-23] to give the approximate maximum volume fraction for a random in-plane case where $C$ is in the range 15-30. Figure 12 shows the values of $V_{f} . L / D$ in these samples are in the range 4-12 with an increasing trend for increasing fibre content. This appears to correlate quite well with the range of values predicted for a 3D random fibre orientation. At first sight this might appear at odds with the high levels of fibre orientation found in these injection moulded samples $[13,16]$. However, it is likely that the many of the important processes leading to reduction of the fibre length take place during the melt plasticization step of the moulding process prior to injection. At this stage of the process a much more random fibre orientation is likely to be present in the melt.

The impact resistance of composite materials is a complex subject where the data often reflects the parameters of the test as much as the structure-performance relationships of the materials under test. One major discriminating factor is whether or not the sample under test has been notched. This fact is reflected in the different trends that we observe in Figures 2-7 between notched and unnotched impact results. From a structureperformance viewpoint composite impact strength appears to be influenced by all of the same parameters as tensile strength, although to differing degrees. Notched impact data generally reflect the energy required to propagate an existing crack through the sample, whereas unnotched impact data will depend on both initiation and propagation energies. The effect of fibres on the propagation of a crack through a thermoplastic matrix is to increase the volume in which energy dissipation can take place. The presence of fibres also increases the number of potential energy absorbing mechanisms in the system. It 
has been proposed that various energy dissipating mechanisms may operate when a discontinuous fibre reinforced composite fractures from an existing notch [18].

Deformation and fracture of the matrix takes place in an area in front of the crack tip. At the same time the applied load, transferred by shear to the fibres, may exceed the strength of the fibre-matrix interface and debonding may occur. Transfer of stress may still be possible to a debonded fibre via frictional forces along the interface. Fibres may fracture if the fibre stress level exceeds the local fibre strength. Fibres that have fractured away from the crack interface will be pulled out of the matrix, which may also involve energy dissipation. There is still some debate over which, if any, of these mechanisms predominates in the process. However, it is clear that fibre volume fraction and length (or aspect ratio) are important factors in all these mechanisms since the volume of the energy absorbing zone will be closely related to the number of fibres which the crack must traverse and the volume around the crack (tip) into which the fibres can distribute the deformation. Furthermore, it is clear from Figures 2 and 3 that the notched impact strength of injection moulded GF-PA6,6 is increased by increasing both the volume fraction and length of the reinforcing fibres. It has recently been discussed $[18,19]$ how the notched impact strength of glass reinforced polypropylene can be modeled by a "rule of mixtures" equation

$U_{c}=V_{f} U_{f x}+V_{m} U_{m} \quad \Rightarrow \quad U_{c}-V_{m} U_{m}=V_{f} U_{f x}$

$\mathrm{U}_{\mathrm{m}}$ is the notched impact strength of the matrix and $\mathrm{U}_{\mathrm{fx}}$ is the contribution to the composite impact strength made by all of the different mechanisms caused by the presence of the fibres. It was further shown that $U_{f x}$ was related to the value of $V_{f} \cdot L / D$. Figure 13 shows the fibre contribution to the notched Izod impact plotted against the value of $\mathrm{V}_{\mathrm{f}} \mathrm{L} / \mathrm{D}$ for the composites in this study. It can be seen that there is a reasonable 
level of correlation in Figure 13 and that the results for the LF and SF samples now appear to fall on the same line. It should be noted that using the notched Charpy results produces a very similar relationship. Given the outcome of the above discussion on the likely inverse relationship between $L / D$ and $V_{f}$ and the maximum value of their product obtainable depending on the average fibre orientation this suggests that there may exist an upper boundary on the fibre contribution to the composite notched impact performance in injection moulded composites.

The influence of test temperature on the results shown in Figures 3-5 from the notched Charpy tests are examined in more detail in Figure 14. Lowering the test temperature from $+80^{\circ} \mathrm{C}$ to $-40^{\circ} \mathrm{C}$ reduces the notched impact resistance of the PA6,6 matrix by $80 \%$. This ductile-brittle transition is well known to be associated with the glass transition temperature of the polymer [24]. The fact that significant additional energy dissipation mechanisms are activated by the addition of fibres is clear in Figure 14. The loss in impact resistance across the same temperature range is reduced to only $40 \%$ for the SF samples and is only $20 \%$ in the case of the LF samples. Not only does this show that additional energy dissipation mechanisms are active but also that the fibre length is a key parameter in these processes. However, it is also clear from Figure 14 that the polymer matrix continues to contribute significantly to the notched impact performance. This can be seen from the fact that the shape of the impact-temperature trend-lines for the SF samples follows that same pattern as that of the extruded polymer whereas the LF results tend to follow a similar shape to the results obtained from the fresh moulded polymer sample.

The results for the unnotched impact dependence shown in Figures 6 and 7 are surprisingly well in line with the results previously published with short fibre 
reinforcement [11-13]. The unnotched impact strength of many thermoplastic polymers is more than an order of magnitude greater than their notched impact strength. In general this ratio is significantly reduced by the addition of fillers or fibres. Such reinforcements can severely reduce the energy required to initiate a critical flaw in the system while concurrently the presence of fibres can significantly increase the resistance to crack propagation (as discussed above). It has been shown that fibre-matrix debonding at the fibre tips can occur well before the failure of a composite under load $[1,2]$. These debonded regions can act singly, or at higher strain levels through multiple interactions, as critical flaws where the size of the flaws can be proportional to the average fibre diameter. Increasing addition of fibres can also lead to an increase in unnotched impact by increasing the composite stiffness and therefore the energy required to achieve the strain levels for such debonding to occur. This notching effect of fibres already reaches its maximum influence at very low fibre contents of the order of magnitude of $2-4 \%$ wt. [13] Consequently, in the normal range of fibre contents found in these composites, the resin properties have only a limited influence on the unnotched impact strength and the composite performance in this type of test is dominated by fibre and interface effects. Despite the fact that the average fibre length is significantly greater in these LF composites there are still a very large number of short fibres and glass particles present in the system due to the significant amount of fibre breakage that occurs during the injection moulding process. It may well be that the level of short fibres and particulates (which may become completely debonded at low strain levels) is still of a sufficiently high level to generate the critical flaw in a LF sample unnotched impact test at a similar level as obtained in a SF sample.

The above discussion relates only to these materials in the dry state. The data in Figure 8 show that boiling water conditioning results in a very different pattern of unnotched 
impact response. The disappearance of the fibre and interface influence on the unnotched impact has previously been noted $[14,15]$ in data from similar experiments on injection moulded SFPA after hydrothermal conditioning in ethylene glycol solution. The boiling water conditioning has already been shown to significantly reduce the Young's modulus, tensile strength and interfacial strength of these composites $[16,17]$. At the same time increases in the tensile elongation to failure are noted. It is also well known that the accompanying water absorption lower the glass transition temperature of the PA6,6 matrix from approximately $70^{\circ} \mathrm{C}$ in the dry state to well below room temperature in the conditioned state [24]. For unreinforced PA6,6 this results in a transition from brittle to ductile failure and a significant increase in the energy dissipated in impact testing. Although this may not translate directly into composite performance it is possible that Figure 8 is showing more evidence of a matrix dominated failure with the reduction in glass transition temperature coupled with the lowering of the stress transfer capability of the interface. As discussed above the unnotched Charpy impact performance of these composites is dominated by the energy required to produce the first critical flaw. Failure of a Charpy specimen is usually initiated through tensile failure on the back side of the bending specimen. Although pendulum impact testing is carried out at much higher rates than tensile testing, it is possible to obtain an estimate of the energy required to break a specimen in a tensile test from a combination of the tensile strength and elongation to failure. Figure 15 shows data from such an analysis plotted versus fibre content for both DaM and boiling water conditioned samples. It can be seen that this analysis reproduces the trends shown in Figures 7 and 8 quite accurately. The DaM samples show a strong dependence on fibre content and fibre diameter, whereas the conditioned samples show little dependence on either parameter and an approximately constant value in the middle of the DaM range.

It was previously noted that there appeared to be similarities between trends observed in the instrumented impact results and those obtained in the pendulum impact tests. These similarities are further examined in Figures 16 and 17. Figure 16 compares the results for unnotched Charpy with the instrumented impact energy to peak load. Whereas Figure 17 compares the notched Charpy results with the instrumented impact total energy. It can be seen that a significant correlation is observed in both the Figures. This 
observation further supports the hypothesis that the unnotched pendulum impact performance of glass-fibre reinforced polyamide is related mainly to the generation of the first critical flaw in the system. In a previous study [12] of the influence of fibre diameter on the mechanical performance of injection moulded short fibre reinforced PA6,6 a loss of unnotched impact of approximately $-6 \%$ per $1 \mu \mathrm{m}$ increase in average fibre diameter in the range of $10-17 \mu \mathrm{m}$ was reported. In Figure 18 the data from these LF samples are examined in a similar manner. The figure shows the diameter dependence of the unnotched Izod impact performance from the LF samples at three fixed fibre contents normalised to the performance level for the $10 \mu \mathrm{m}$ diameter samples. The data is compared with a previously published study of injection moulded SFPA at 33\% fibre content. It is clear from this figure that the LF samples exhibit a very similar unnotched impact inverse dependence on average fibre diameter as previously observed with SF samples although the increased fibre length has mitigated the sensitivity on fibre diameter to some extent with an average slope in Figure 18 of $-4 \%$ per $1 \mu \mathrm{m}$ increase in fibre diameter.

\section{Conclusions}

This study of injection moulded long glass-fibre reinforced polyamide 6,6 composites has revealed that the average residual fibre length of all LFPA samples exhibited an inverse dependence on fibre content at fixed fibre diameter and a strong dependence on the average fibre diameter at fixed fibre content. These dependencies resulted in an invariable residual fibre aspect ratio for long fibre samples prepared with the same fibre content and different fibre diameters. The product of the residual fibre aspect ratio and the fibre volume fraction fell in the range of 4-12 indicative of the fibre aspect ratio being limited by a maximum packing fraction of fibres with a 3D random fibre 
orientation at some point during the melt processing. The fibre length and content did have a significant effect on composite notched impact but fibre diameter did not. Fibre diameter and content did have a significant effect on composite unnotched impact but fibre length did not. In instrumented multiaxial impact testing the total energy value followed similar trends to notched impact, the energy to first peak followed the same trends as unnotched impact. Unnotched impact performance of these composites lost all dependence of fibre content, length and diameter after boiling water conditioning. Analysis of the notched impact data provided more evidence that fibre packing considerations in injection moulded thermoplastics results in an upper limit to the value of the product of the fibre volume fraction and aspect ratio, resulting in some kind of upper boundary on the fibre contribution to the composite notched impact performance. The LFPA samples exhibited a very similar unnotched impact dependence on average fibre diameter as previously observed with SFPA samples.

\section{Acknowledgement}

The author gratefully acknowledges the support of Owens Corning Science and Technology with the preparation and testing of the materials used in this study. 


\section{References}

1. Sato N, Kurauchi T, Sato S, and Kamigaito O. Mechanism of fracture of short glass fibre-reinforced polyamide thermoplastic. J. Mater. Sci. 1984:19;1145-1152

2. Sato N, Kurauchi T, Sato S, and Kamigaito O. Reinforcing mechanism by small diameter fiber in short fiber composites. J. Compos. Mater. 1998:22;850-873

3. Horst JJ, and Spoormaker JL. Fatigue fracture mechanisms and fractography of short-glass fibre-reinforced polyamide 6. J. Mater. Sci. 1997:32;3641-3651.

4. Akay M, Barkley D. Fibre orientation and mechanical behaviour in reinforced thermoplastic injection mouldings. J.Mater. Sci. 1991;26:2731-42

5. Laura DM, Keskkula H, Barlow JW, Paul DR. Effect of glass fiber surface chemistry on the properties of glass fiber reinforced, rubber-toughened nylon 6 . Polymer 2002:43;4673-4687

6. Thomason JL. The influence of fibre properties of the performance of glass-fibrereinforced polyamide 6,6. Comp. Sci. Tech. 1999;59:2315-2328.

7. Hassan A, Yahya R, Yahaya AH, Tahir ARM, Hornsby PR. Tensile, Impact and Fiber Length Properties of Injection-molded Short and Long Glass Fiber-reinforced Polyamide 6,6 Composites. J.Reinforced Plastics and Composites 2004;23:969-986.

8. Carlson E, Nelson K. Nylon under the hood: history of innovation. Automotive Engineering 1996;104:84-89.

9. Guyot H. VERTON in Long Fibres. Plast. Mod. Elastom. 1985;37(5):44-45.

10. Toll S, Aronsson C-G. Notched Strength of Long- and Short-Fibre Reinforced Polyamide. Comp. Sci. Tech. 1992;46:43-54.

11. Belbin GR, Staniland PA. Advanced Thermoplastics and their Composites. Philosophical Transactions of the Royal Society of London. Series A, Mathematical and Physical Sciences. 1987;322:451-464. 
12. Bailey RS, Davies M, Moore DR. Processing property characteristics for long glass fibre reinforced polyamide. Composites 1989;20:453-460.

13. Thomason JL. Structure-property relationships in glass reinforced polyamide: 1) The effect of fibre content. Polym.Composites 2006;27:552-562.

14. Thomason JL. Structure-property relationships in glass reinforced polyamide: 2) The effects of average fibre diameter and diameter distribution. Polym.Composites 2007;27:331-343.

15. Thomason JL. Structure-property relationships in glass reinforced polyamide: 3 ) Effects of hydrolysis ageing on the dimensional stability and performance of short glass-fibre reinforced Polyamide 66. Polym.Composites 2007;27:344-354.

16. Thomason JL. The influence of fibre length, diameter and concentration on the modulus of glass-fibre reinforced polyamide 6,6. Compos A 2008;39:1732-1738.

17. Thomason JL. The influence of fibre length, diameter and concentration on the strength and strain to failure of glass-fibre reinforced polyamide 6,6. Compos A 2008;39:1618-1624.

18. Thomason JL and Vlug MA. The influence of fibre length and concentration on the properties of glass fibre reinforced polypropylene: 4) Impact Properties. Composites 1996;28A:277-288.

19. Thomason J.L. The influence of fibre length and concentration on the properties of glass fibre reinforced polypropylene: 5) Injection moulded long and short fibre PP', Compos A 2002;33:1641-1652.

20. Williams JCL, Watson SJ and Boydell P. Factors affecting the properties of Nylons. In: Kohan MI, editor. Nylon Plastics Handbook. Munich: Hanser, 1995. p.294.

21. Gibson AG and Payne DJ. Flexural and impact strength improvement in injected moulded SMC. Composites 1989;20:151-158. 
22. Evans KE and Gibson AG. Prediction of the maximum packing fraction achievable in randomly oriented short-fibre composites. Compos.Sci.Technol. 1986;25:149162.

23. Toll S, Ericson A, Zuber A. and Manson JE. Packing of discontinuous fibres.

Proceedings of ICCM-9, Vol III, 391-397, Woodhead Publishing Limited, Cambridge, UK 1993,

24. Keskkula H and Paul DR. Toughened Nylons In: Kohan MI, editor. Nylon Plastics Handbook. Munich: Hanser, 1995. p.414-430. 


\section{List of Figures}

Figure 1 Residual weight average fibre length versus fibre content

Figure 2 Notched Izod impact versus fibre content

Figure 3 Notched Charpy impact versus fibre content

Figure $4-40^{\circ} \mathrm{C}$ Notched Charpy impact versus fibre content

Figure $5+80^{\circ} \mathrm{C}$ Notched Charpy impact versus fibre content

Figure 6 Unnotched Izod impact versus fibre content

Figure 7 Unnotched Charpy impact versus fibre content

Figure 8 Unnotched Charpy impact after 24 hour boil versus fibre content

Figure 9 Instrumented impact energy to first peak versus fibre content

Figure 10 Instrumented impact total versus fibre content

Figure 11 Residual fibre aspect ratio versus fibre content

Figure $12 \mathrm{~V}_{\mathrm{f} . \mathrm{L} / \mathrm{D} \text { versus fibre content }}$

Figure 13 Fibre contribution to notched Charpy impact

Figure 14 Influence of temperature on notched Charpy impact performance

Figure 15 Tensile energy to failure versus fibre content

Figure 16 Unnotched Charpy versus Instrumented impact energy to first peak

Figure 17 Notched Charpy versus Instrumented impact total energy

Figure 18 Normalised unnotched Izod impact vs Average fibre diameter 


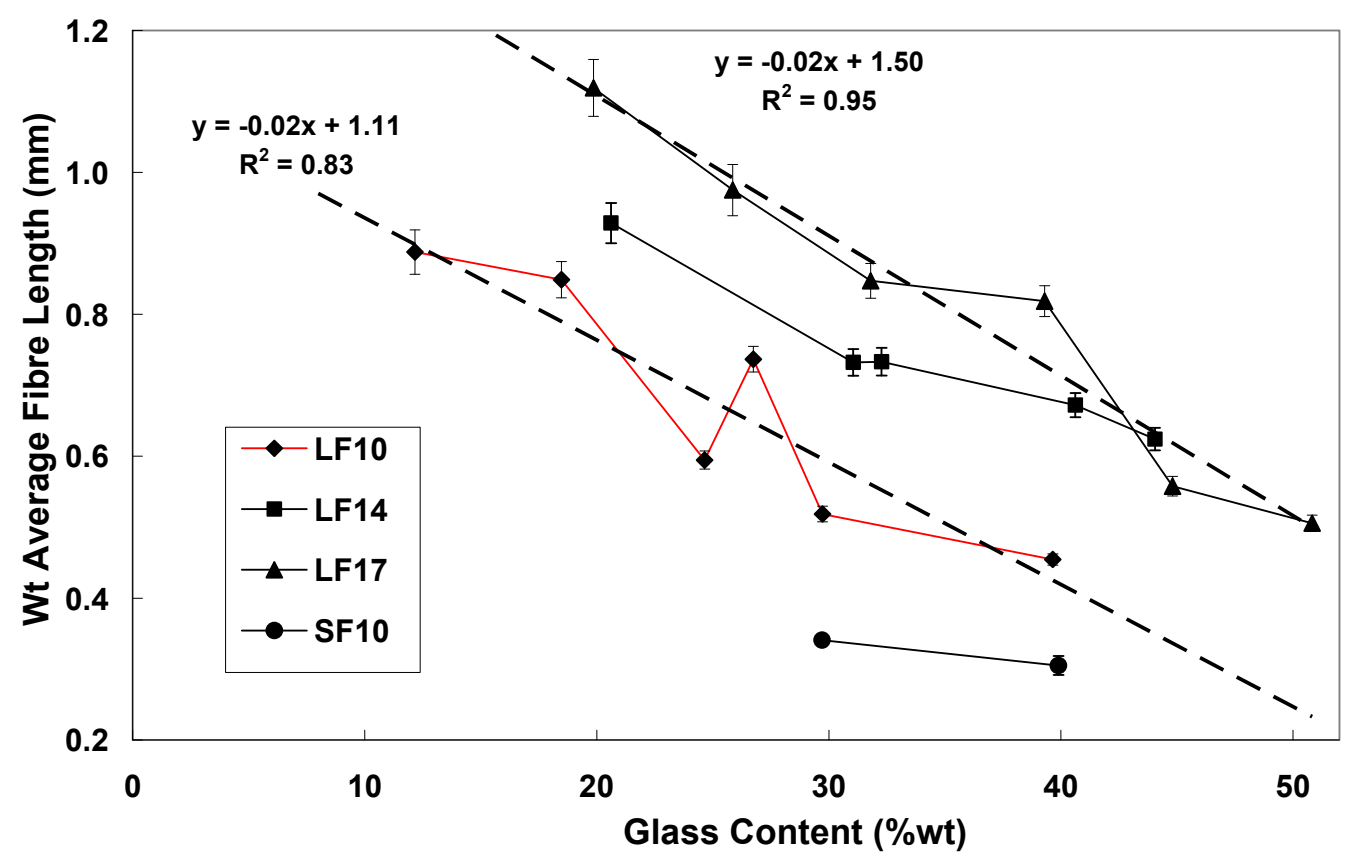

Figure 1 Residual weight average fibre length versus fibre content

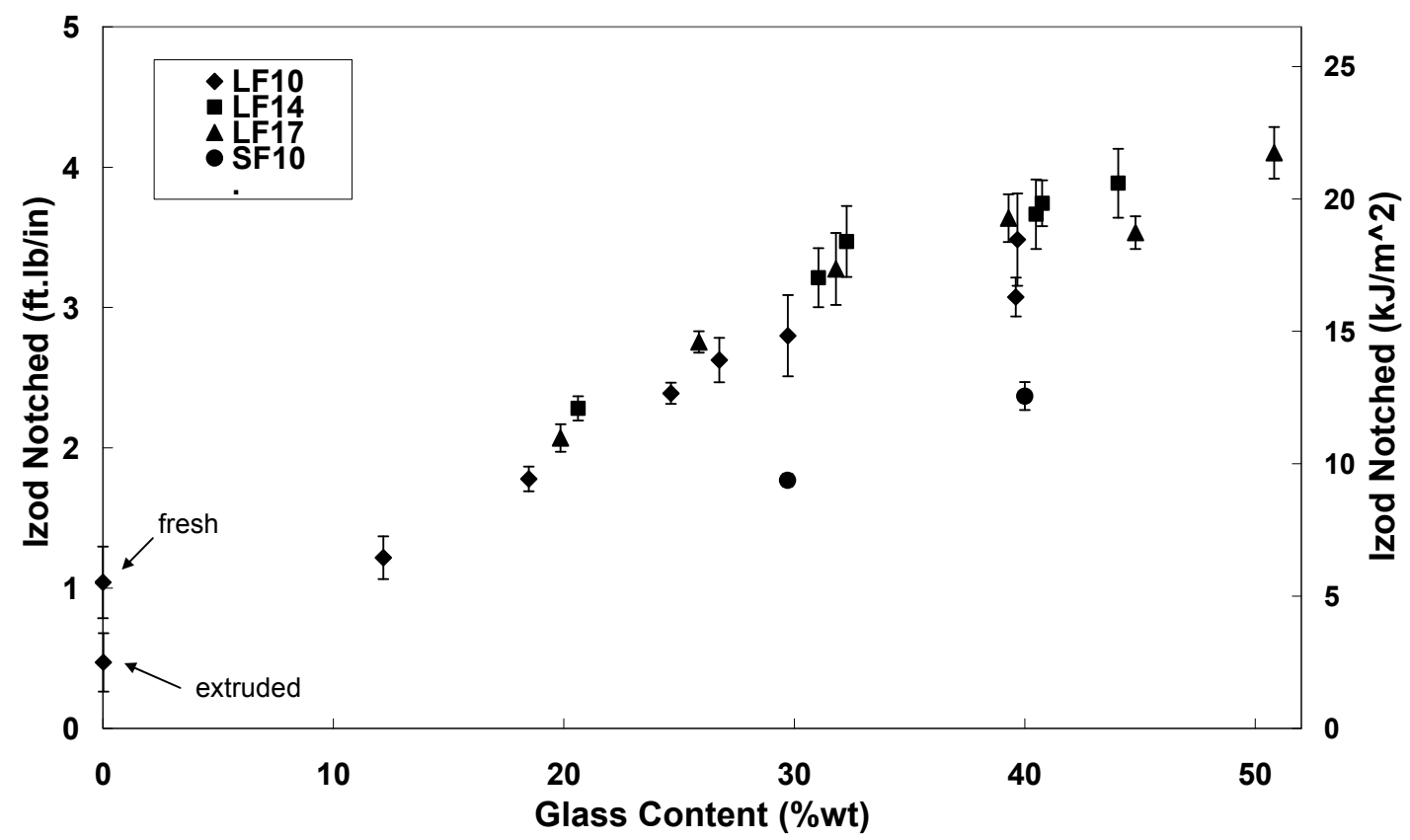

Figure 2 Notched Izod impact versus fibre content 


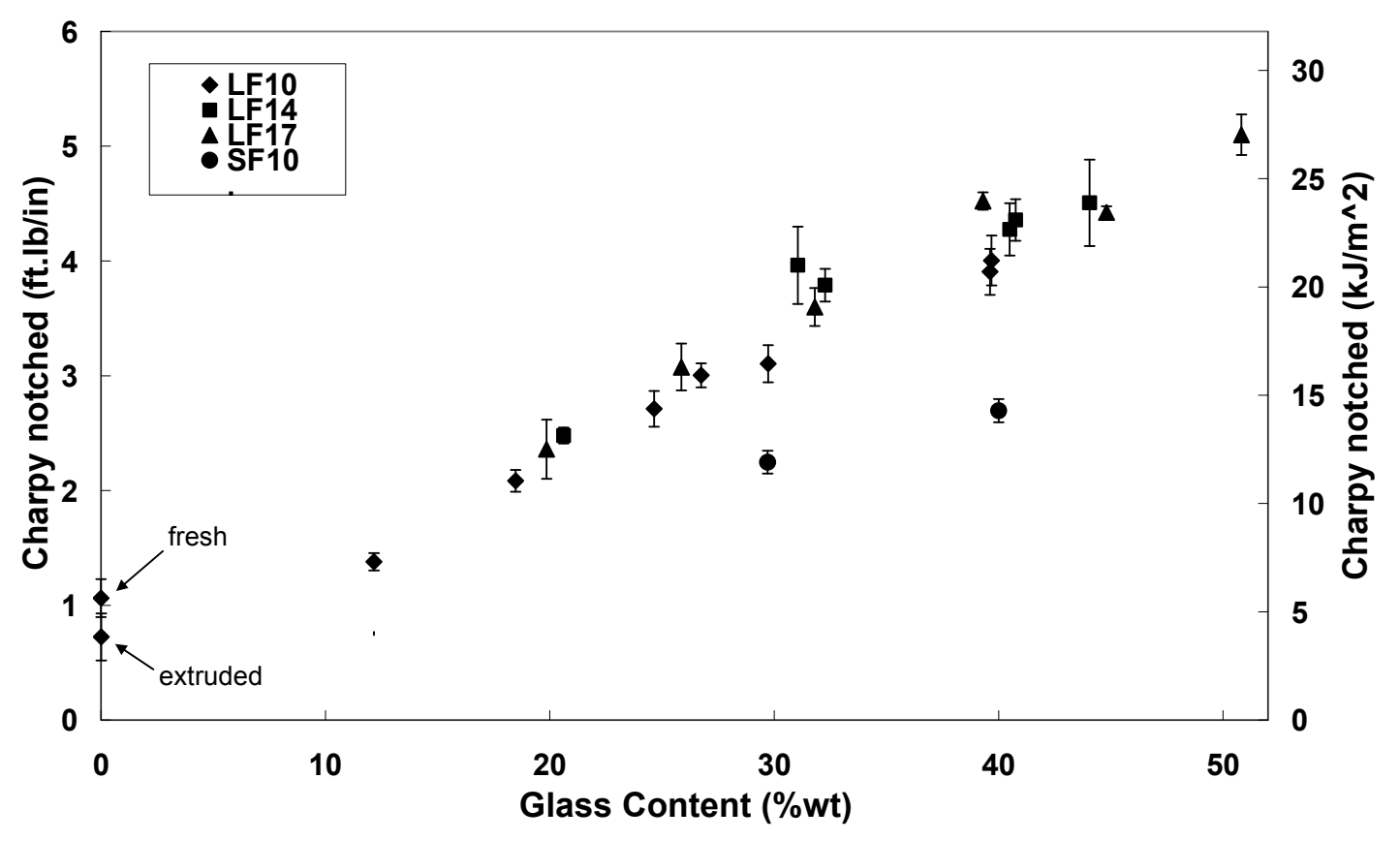

Figure 3 Notched Charpy impact versus fibre content

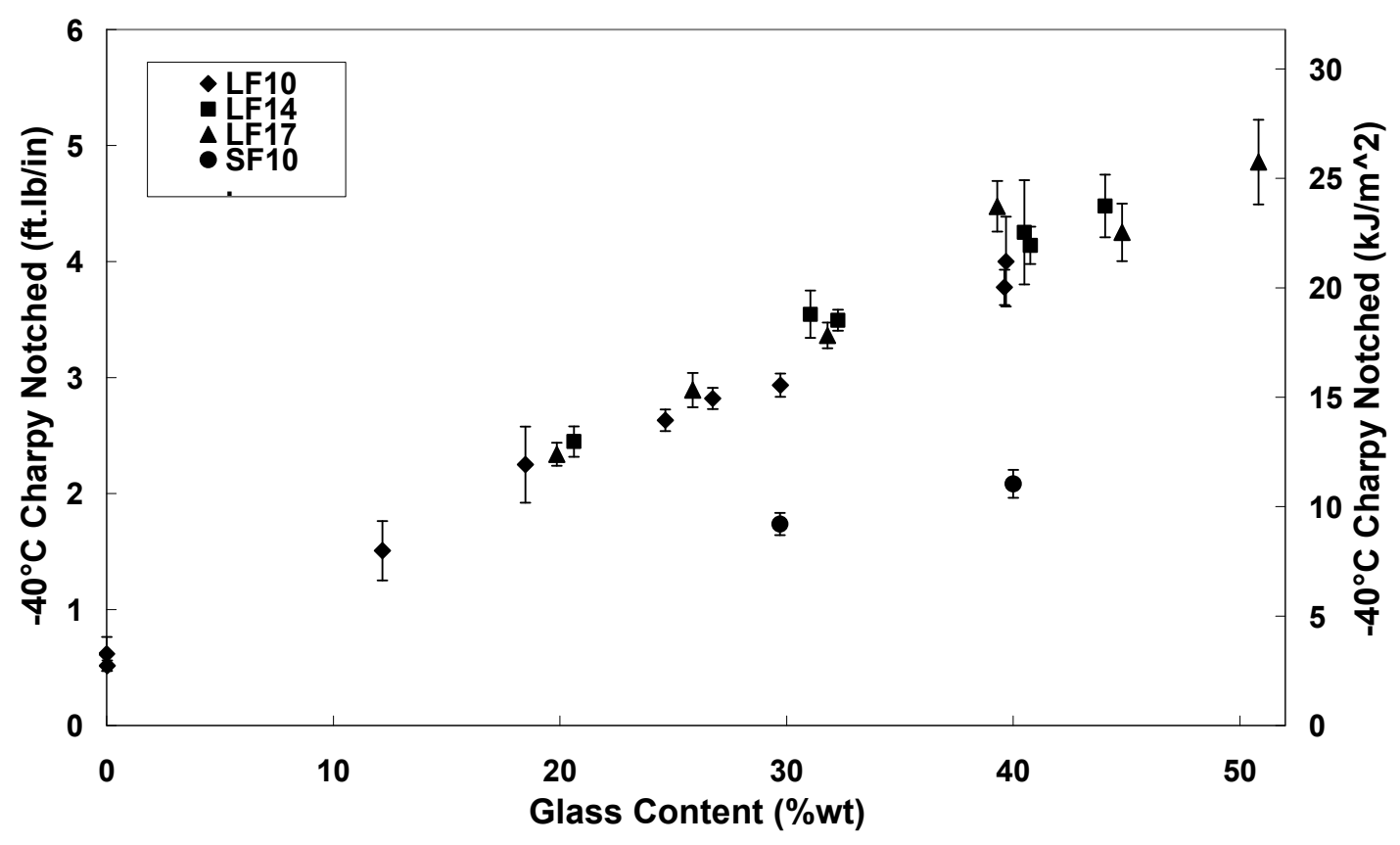

Figure $4-40^{\circ} \mathrm{C}$ Notched Charpy impact versus fibre content 


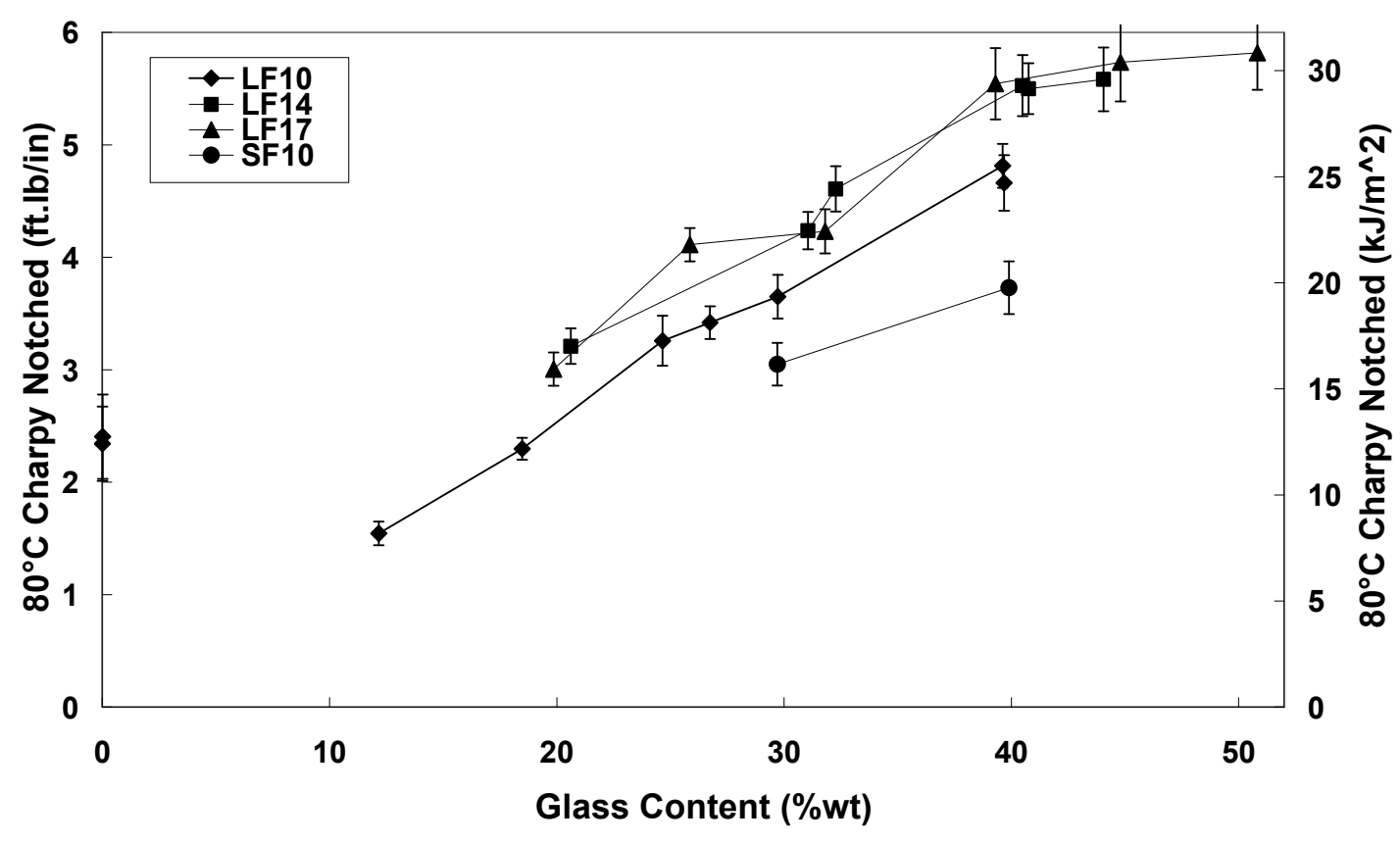

Figure $5+80^{\circ} \mathrm{C}$ Notched Charpy impact versus fibre content

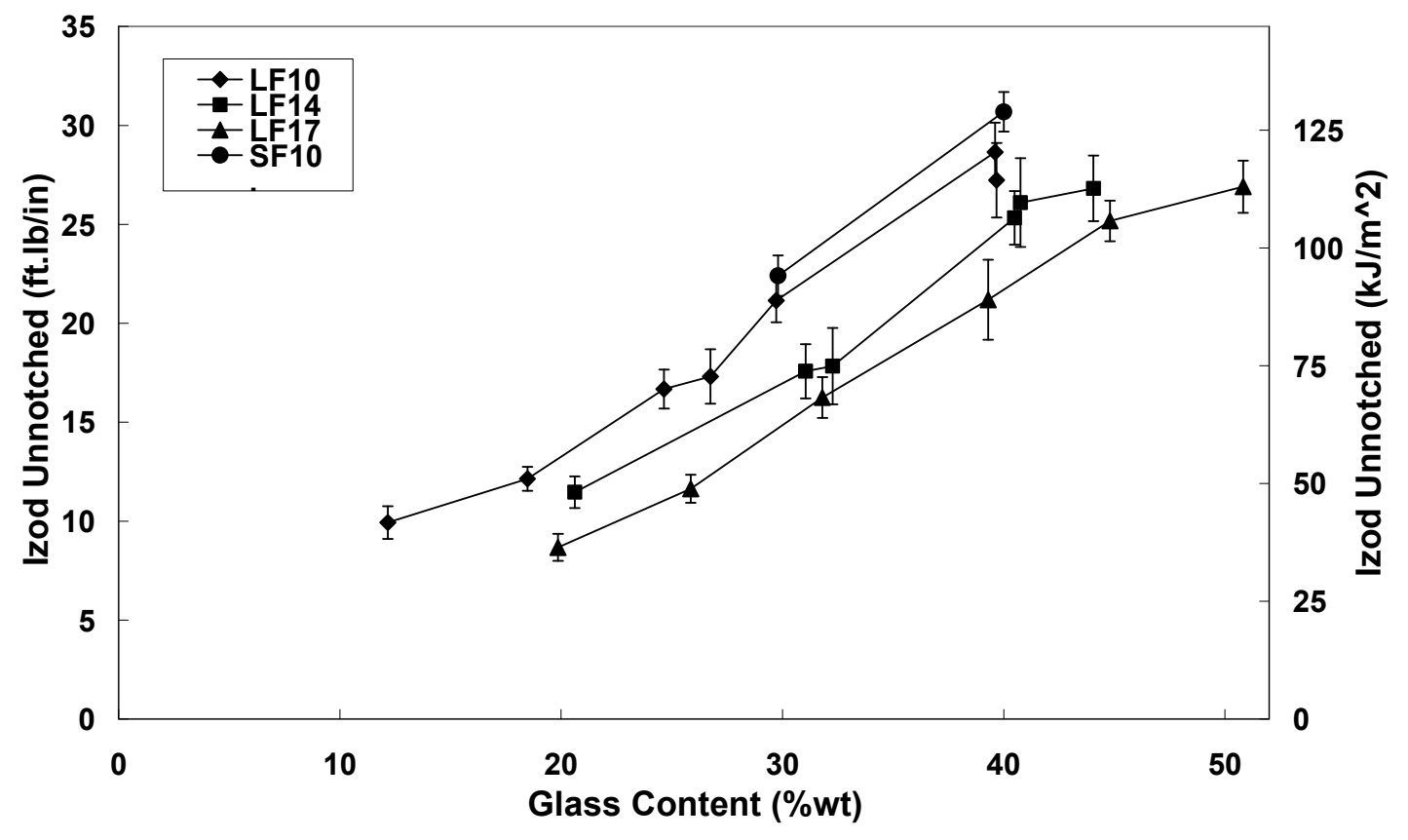

Figure 6 Unnotched Izod impact versus fibre content 


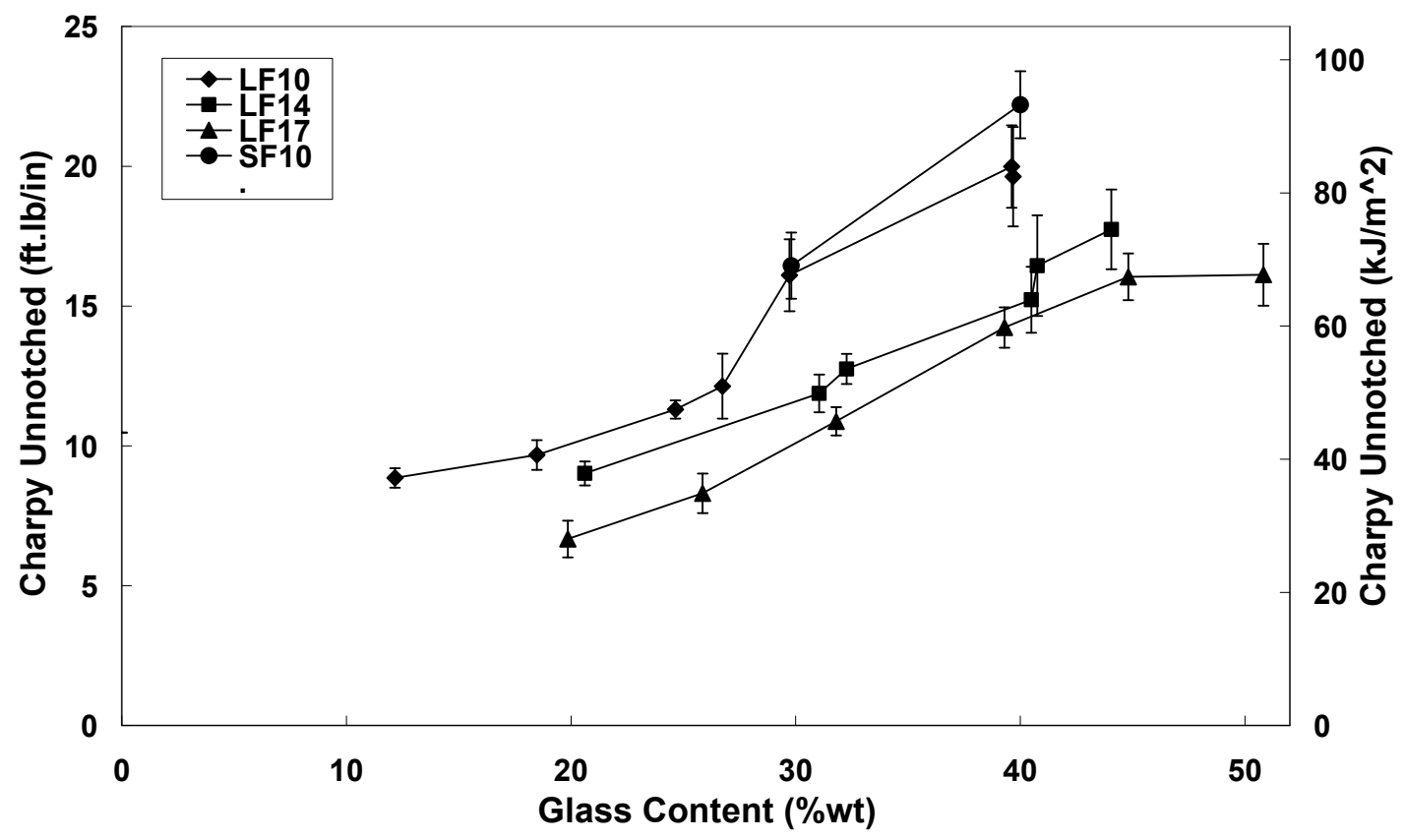

Figure 7 Unnotched Charpy impact versus fibre content

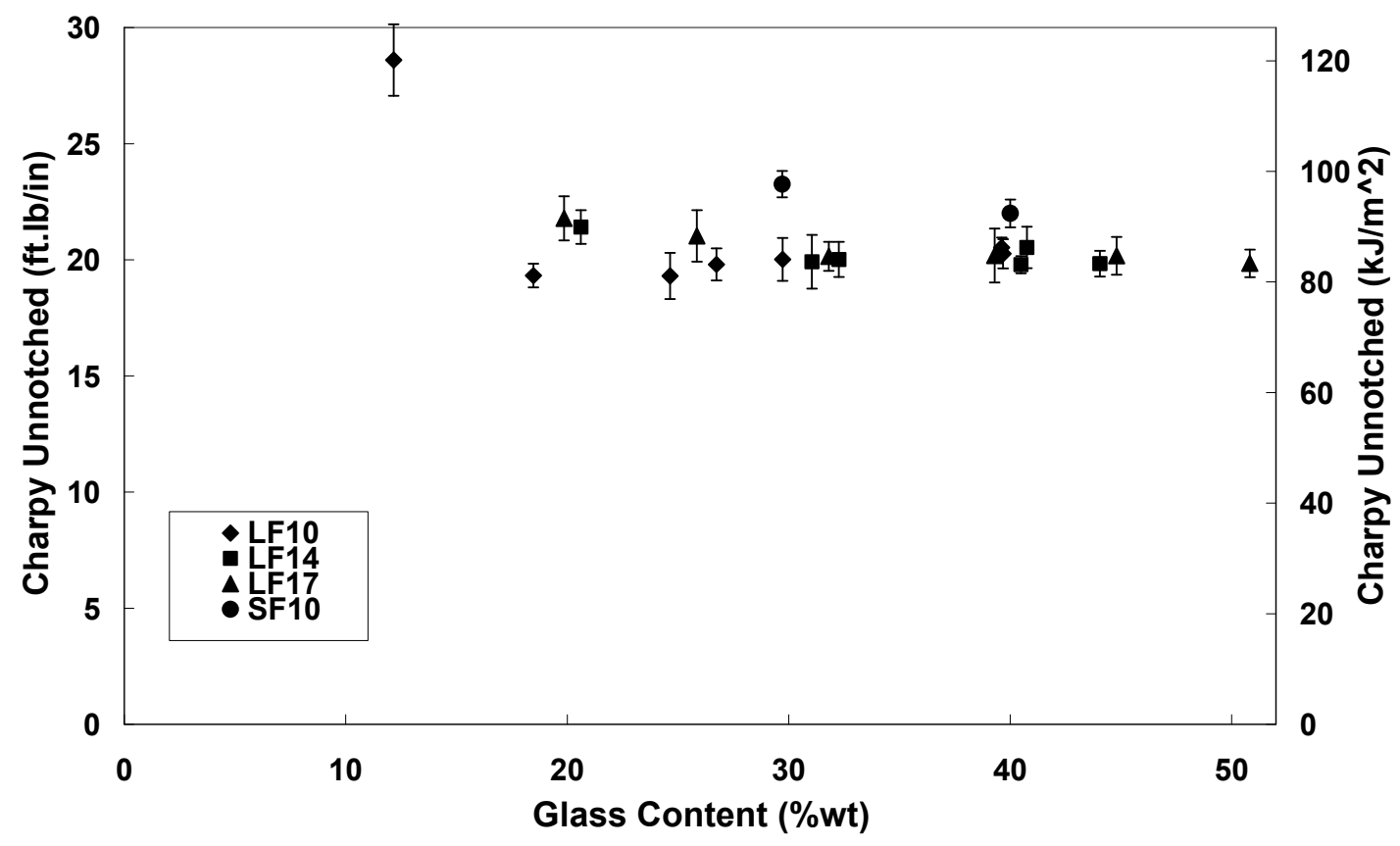

Figure 8 Unnotched Charpy impact after 24 hour boil versus fibre content 


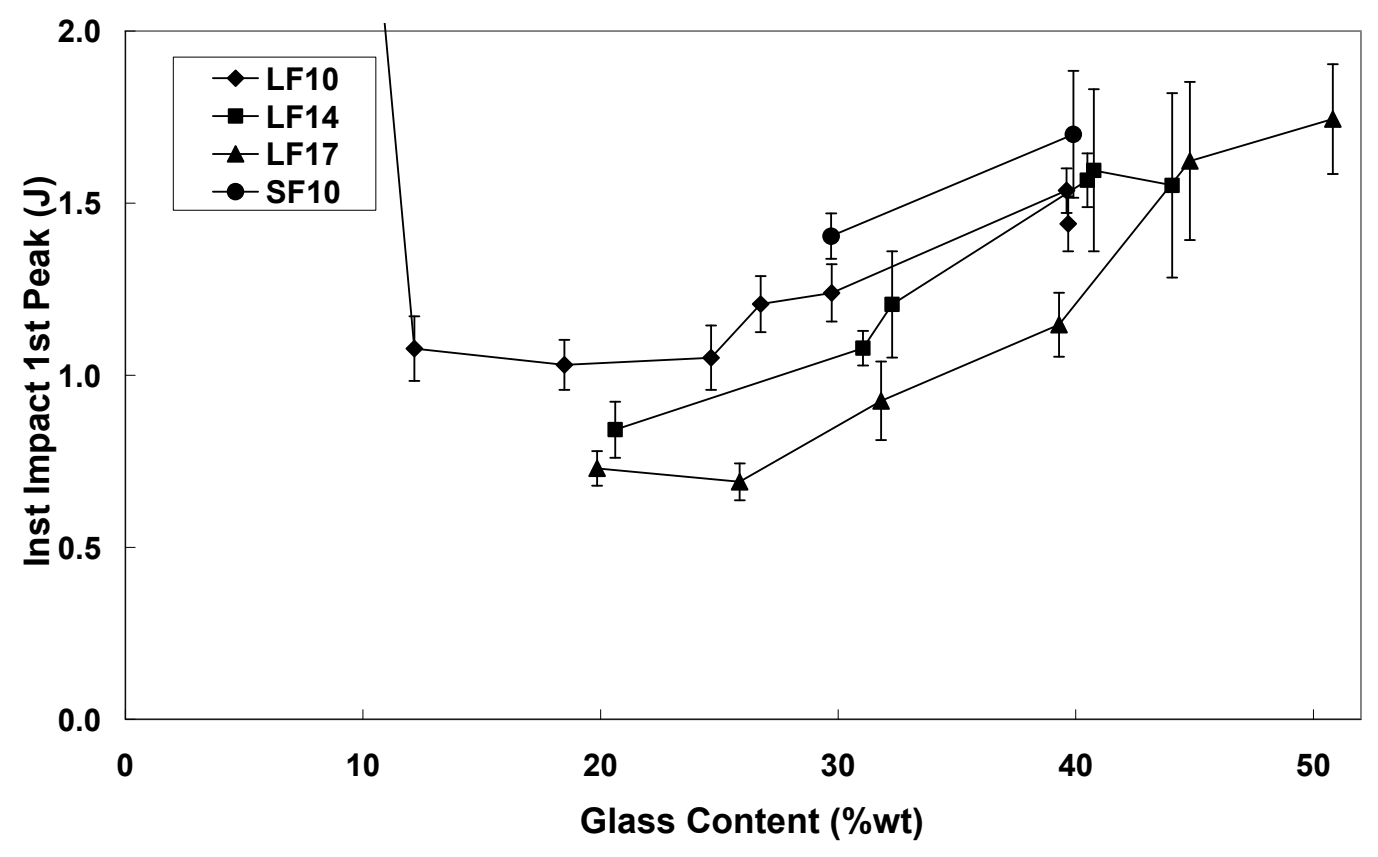

Figure 9 Instrumented impact energy to first peak versus fibre content

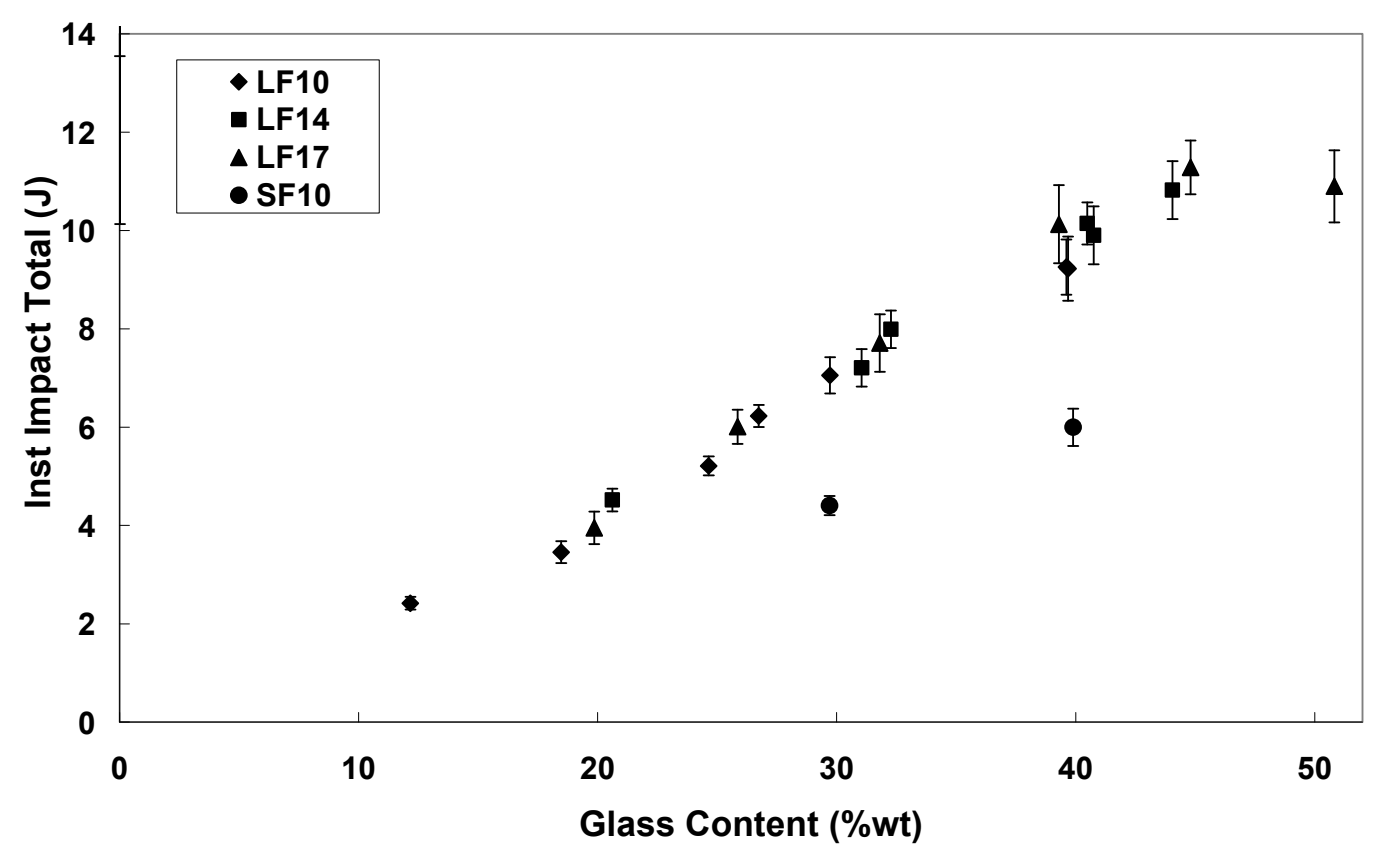

Figure 10 Instrumented impact total versus fibre content 


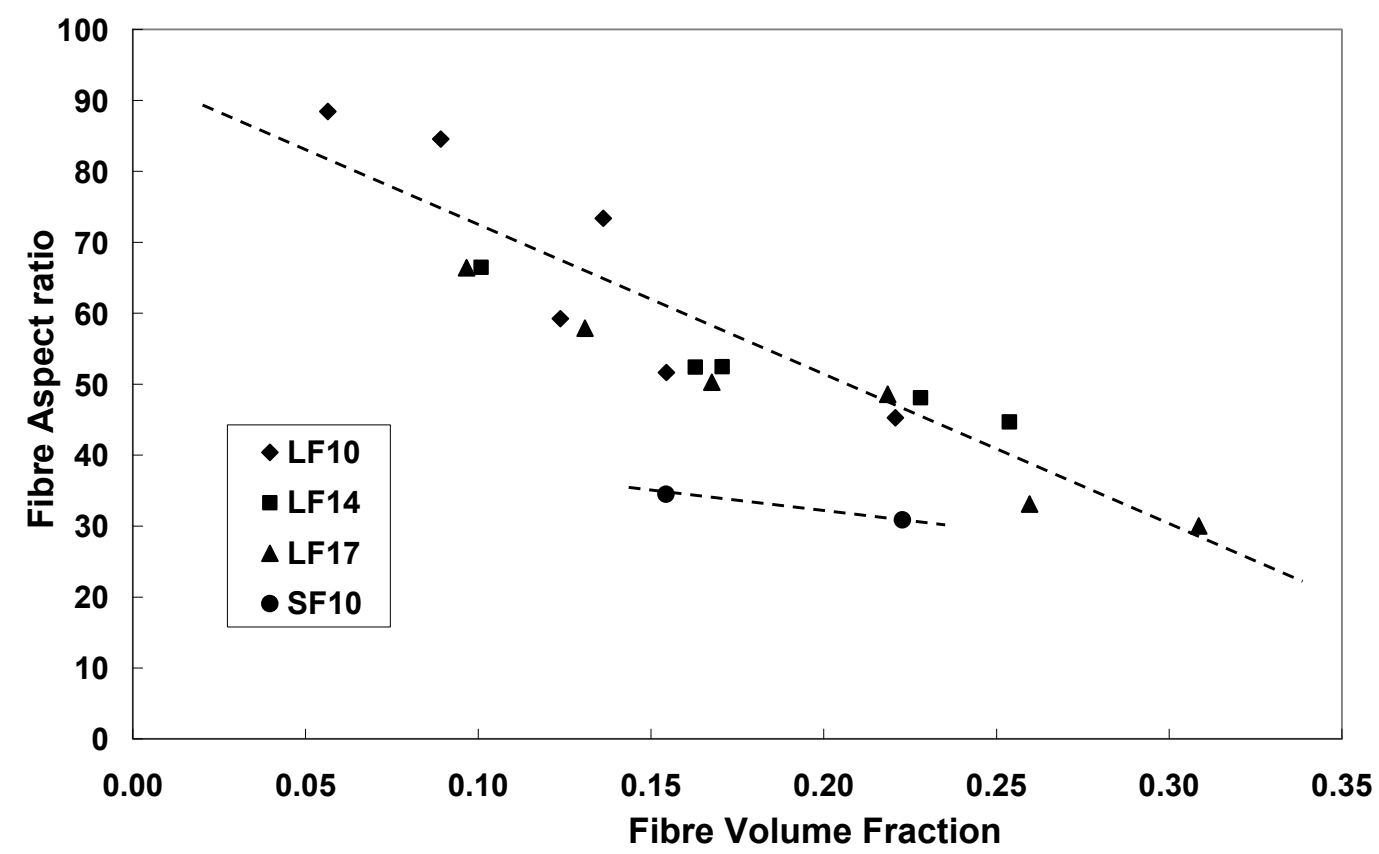

Figure 11 Residual fibre aspect ratio versus fibre content

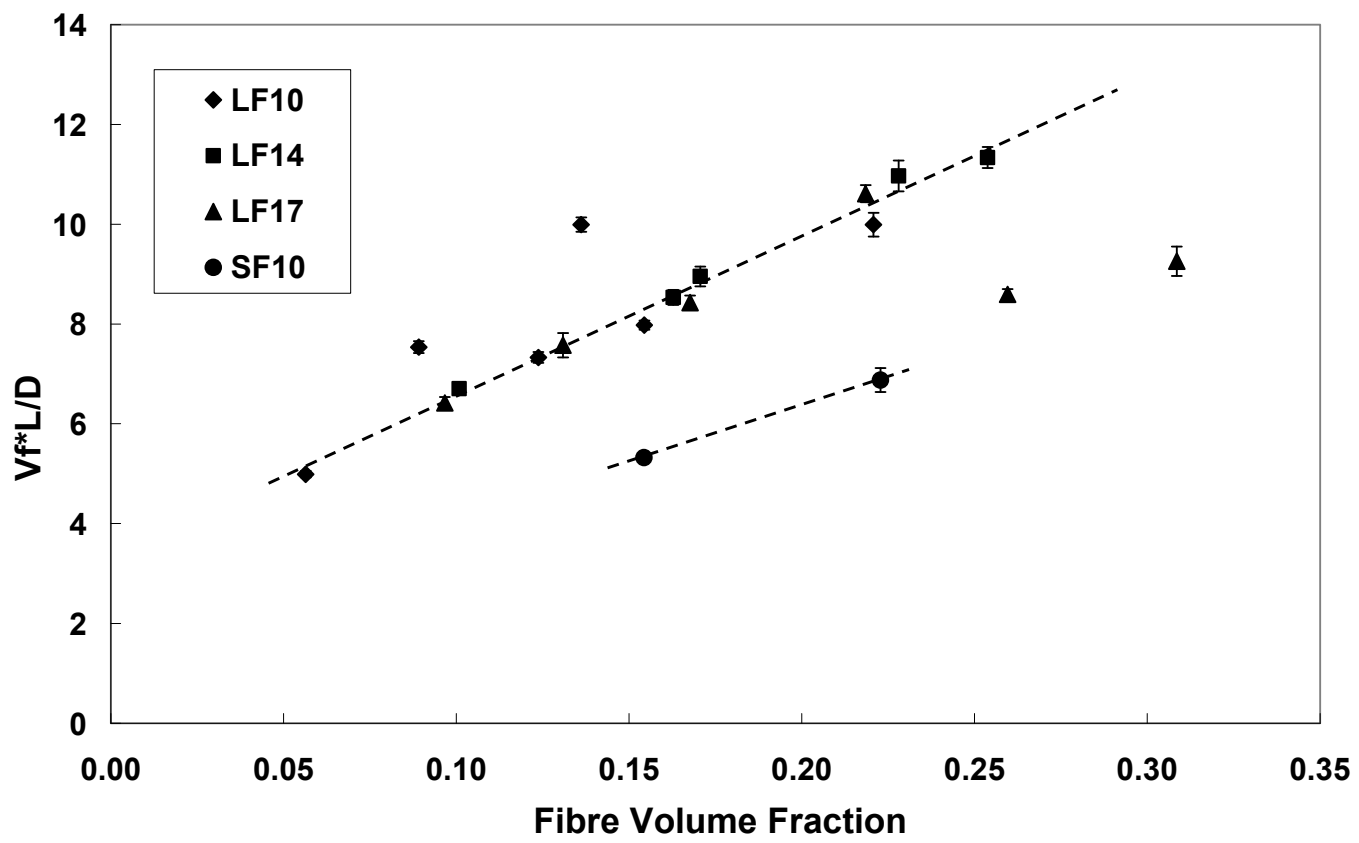

Figure $12 \mathrm{~V}_{\mathrm{f} . \mathrm{L} / \mathrm{D} \text { versus fibre content }}$ 


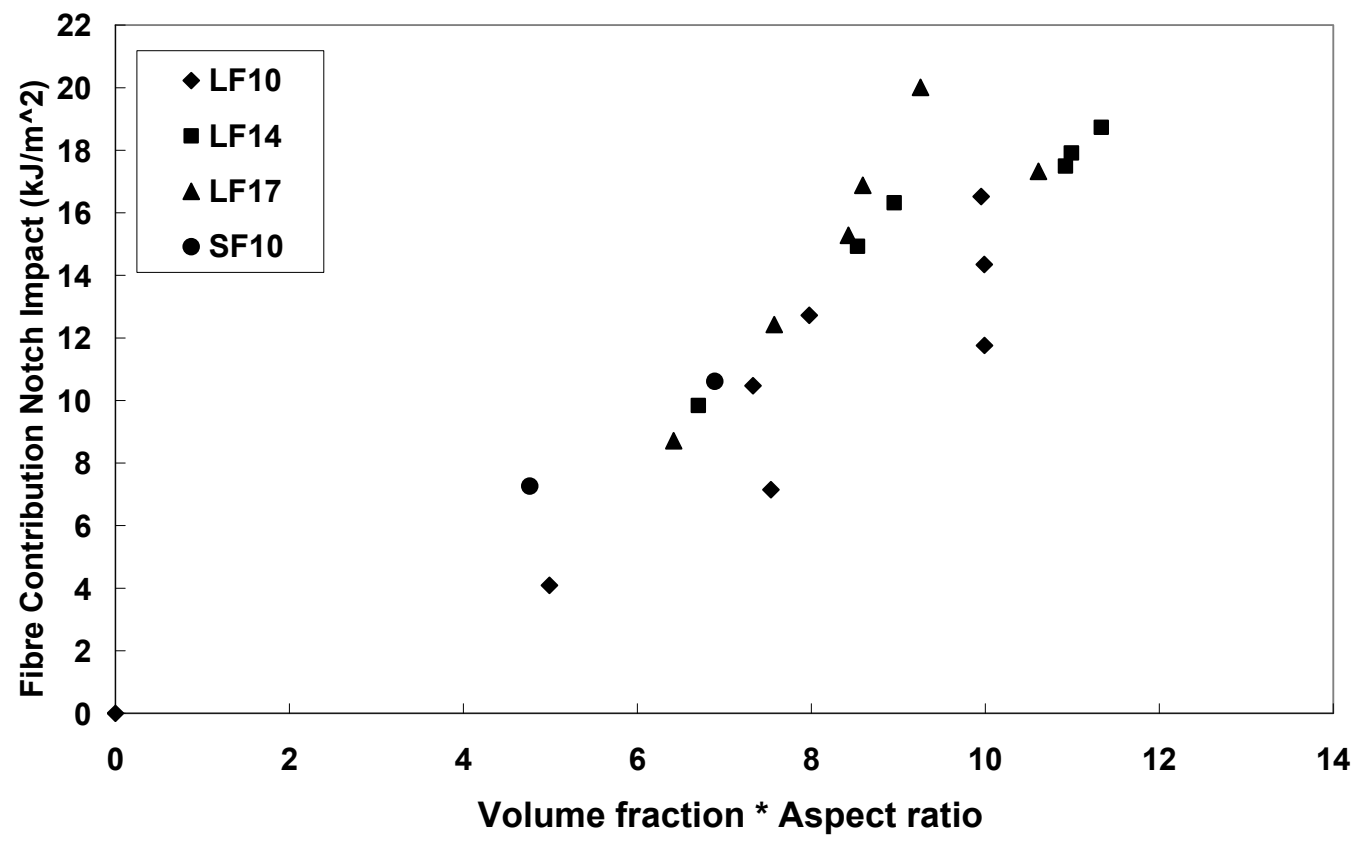

Figure 13 Fibre contribution to notched Charpy impact

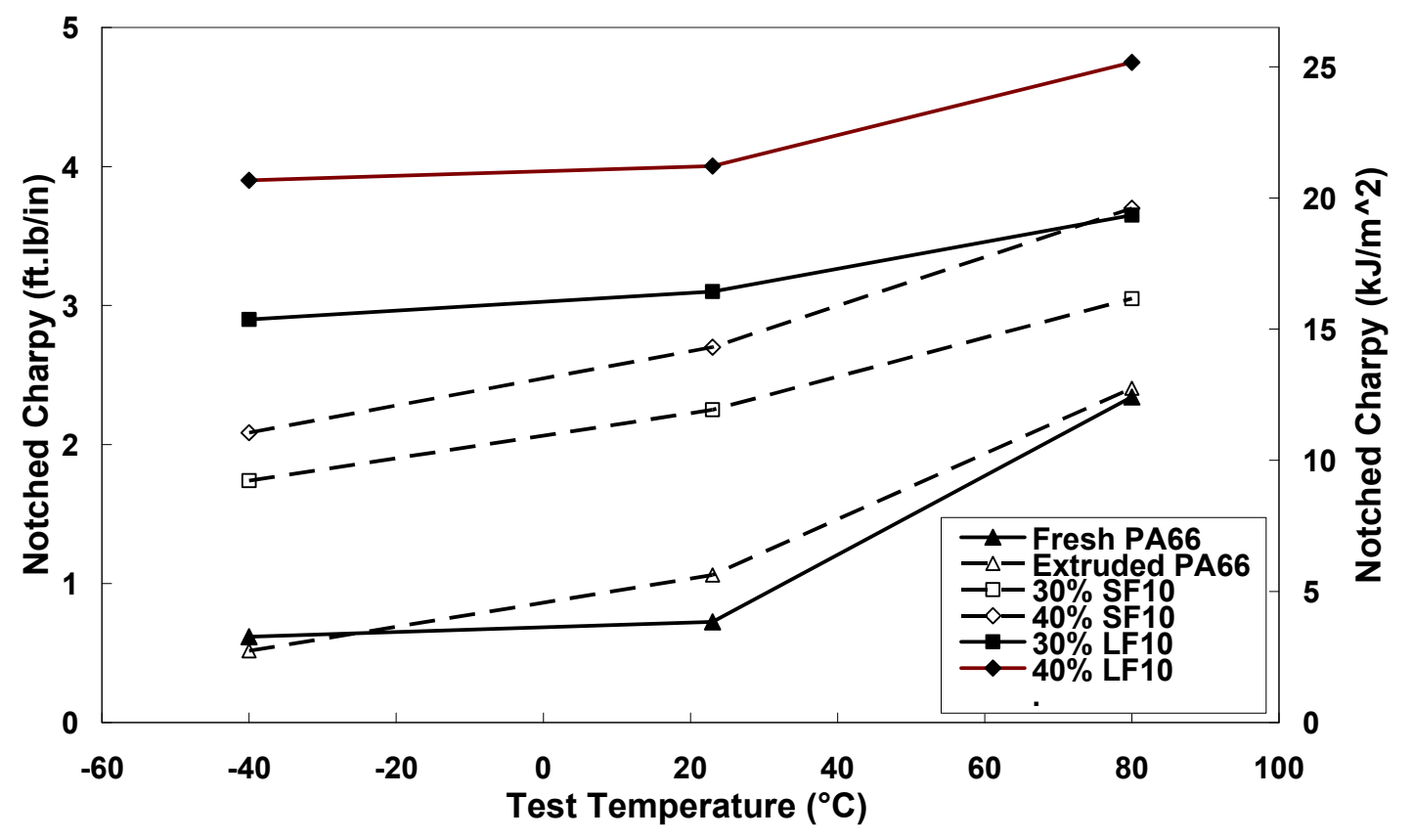

Figure 14 Influence of temperature on notched Charpy impact performance 


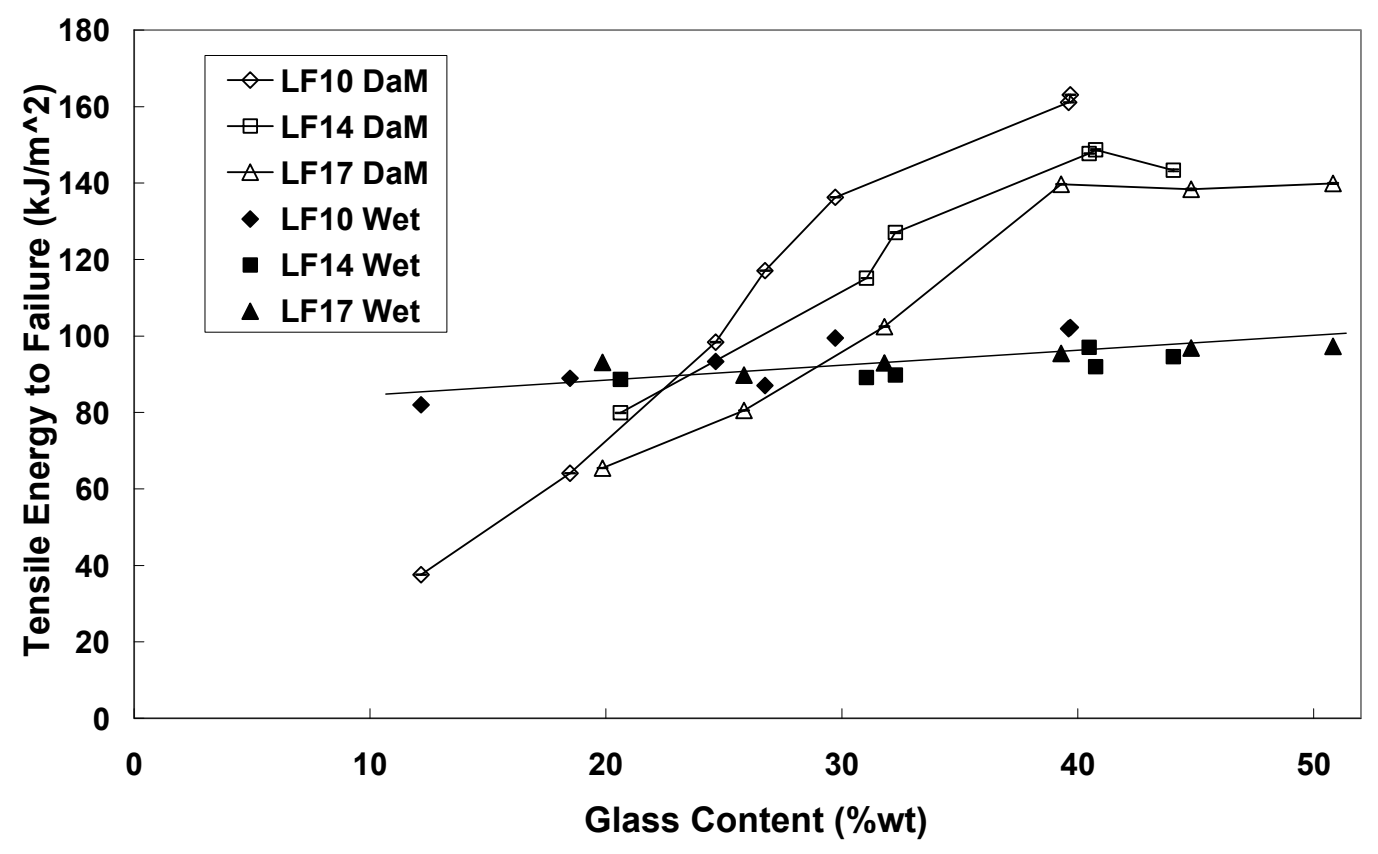

Figure 15 Tensile energy to failure versus fibre content

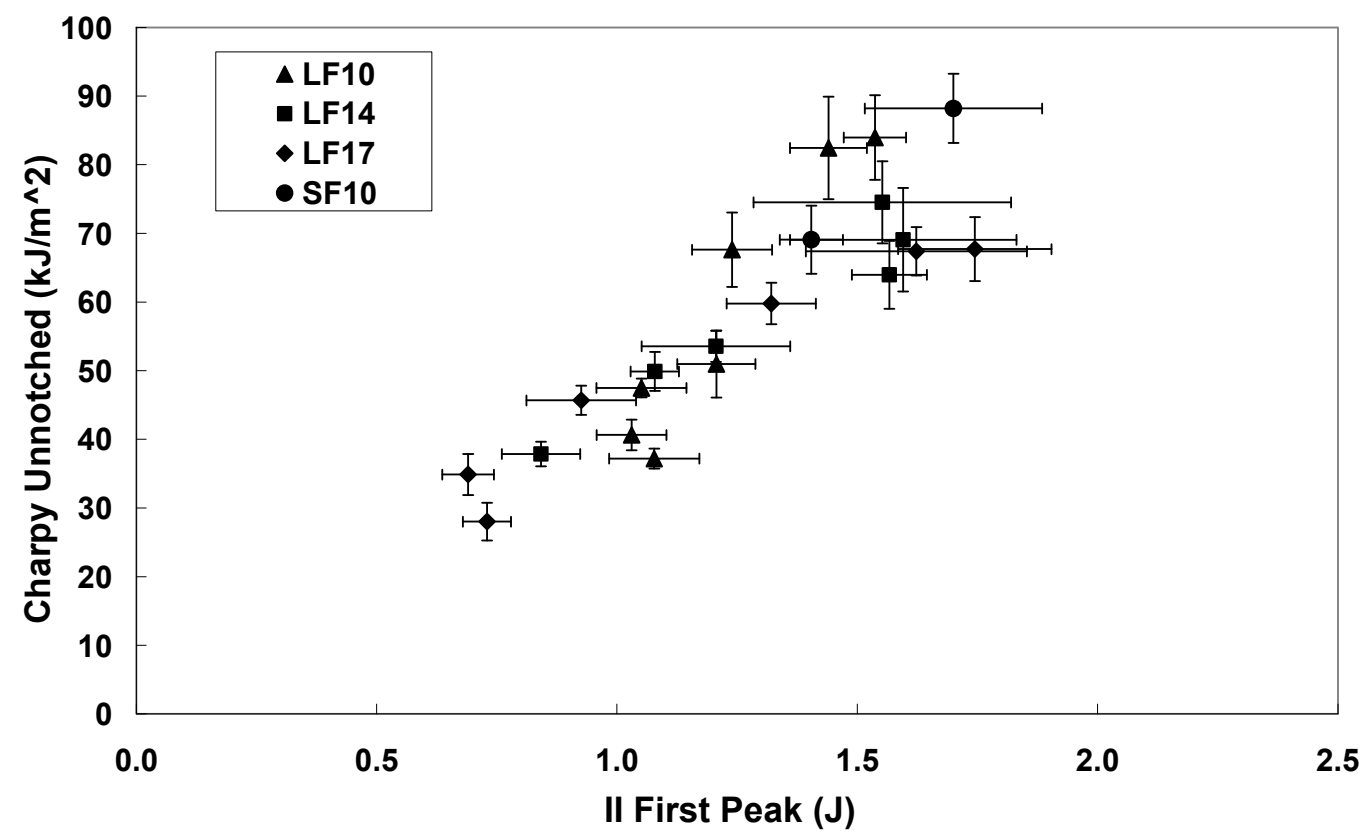

Figure 16 Unnotched Charpy versus Instrumented impact energy to first peak 


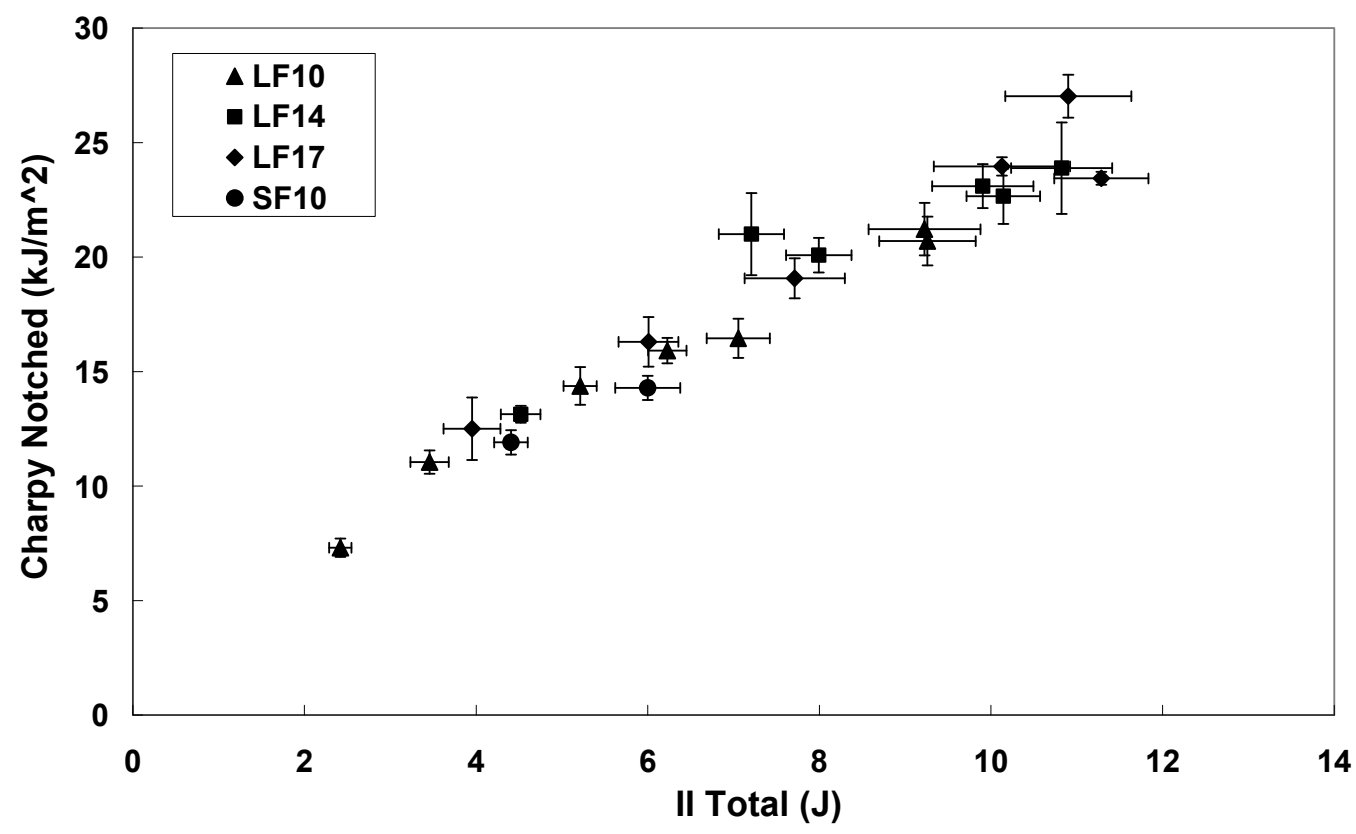

Figure 17 Notched Charpy versus Instrumented impact total energy

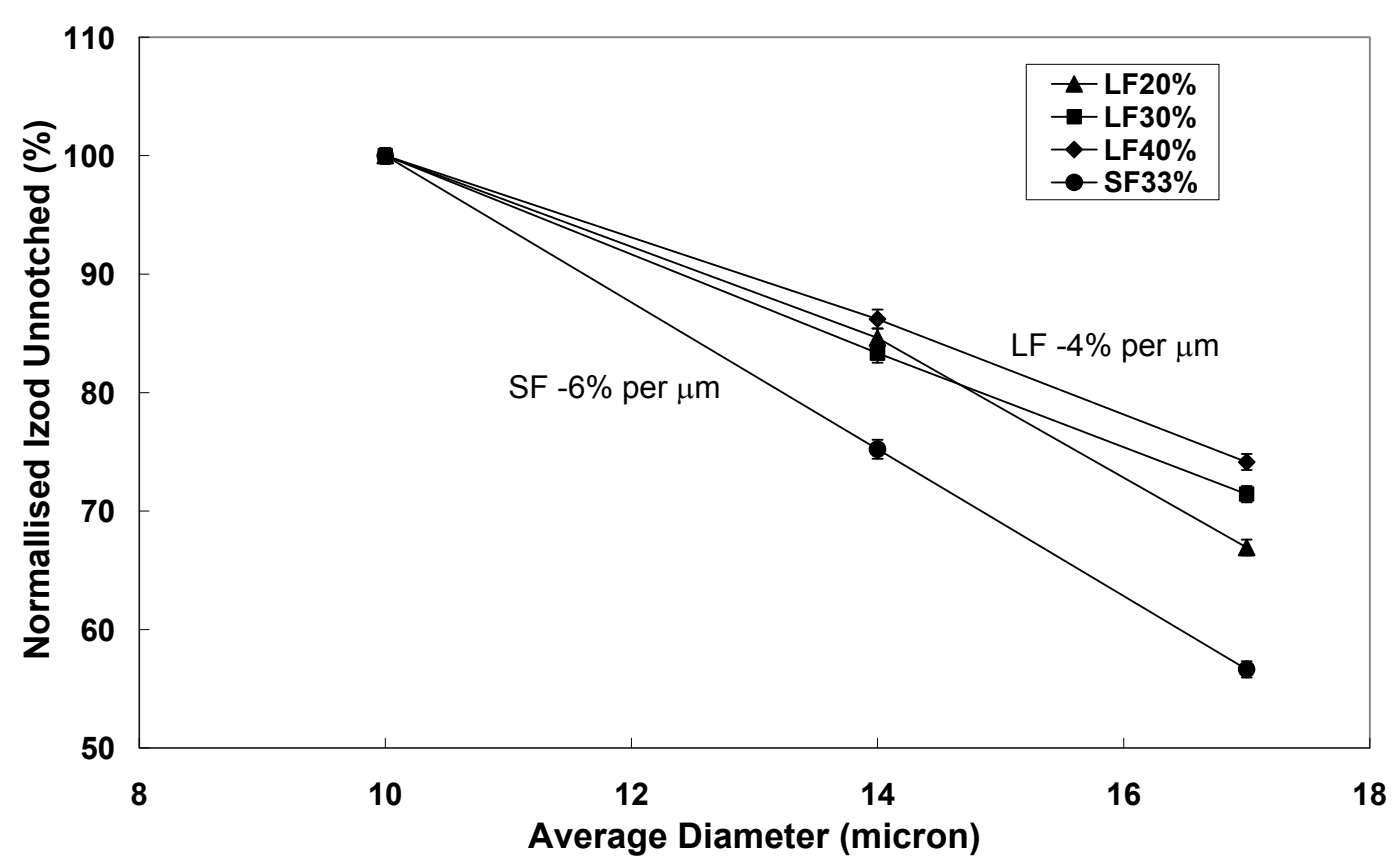

Figure 18 Normalised unnotched Izod impact vs Average fibre diameter 